\title{
Force analysis for pure rolling movable teeth transmission
}

\author{
Yali $\mathrm{Yi}^{\mathrm{a}}$ and Yameng $\mathrm{Ji}$ \\ College of Mechanical Engineering, Yanshan University, Qinhuangdao, 066004, China
}

\begin{abstract}
Based on the principle of sliding and rolling substitution, combined movable tooth design, approaches were presented to eliminate the slip phenomenon, ensure the pure rolling contact of meshing pairs and realize pure rolling movable teeth transmission. According to the coordinate condition of elastic deformation, force analysis was conducted on the meshing pairs of rolling movable teeth transmission. The mathematical model of meshing force was established to analyze the meshing force between shock wave cam, central wheel and movable tooth, the engagement position of maximum contact force was determined, which can provide reference for the further strength check and structure optimization design.
\end{abstract}

Keywords: movable teeth transmission; pure rolling; force analysis.

\section{Introduction}

A new type of movable teeth transmission, pure rolling movable teeth transmission, is proposed after roller movable teeth transmission in[1-2]. With the rapid development of industrial machinery, the dynamic performance of transmission mechanism is improved gradually, the range of transmission ratio is expended increasingly [3] and the multi path or speed transmission is realized. This is such that promoted the development and research of two-stage drive in [4-5], especially the type synthesis and design of two-stage movable tooth transmission are proposed in [6]. Meanwhile, the rolling tooth transmission is planar multi-tooth meshing and belongs to statically indeterminate problem that is complex stress state in [7-8]. In this paper, rolling movable teeth transmission dynamics model is established with deformation coordination equation and meshing force of movable teeth meshing pair is analyzed and calculated. Besides, according to the Hertz contact theory, the contact stress of meshing pairis analyzed. This is such that played a guiding role for analysis and synthesis of the existing roller movable teeth transmission and development of a new type pure rolling movable teeth transmission.

\section{The principle of pure rolling movable teeth transmission}

The pure rolling movable teeth transmission consist of a shock wave cam $\mathrm{H}$, an active tooth frame $\mathrm{K}$, a center wheel $\mathrm{K}$ and some movable tooth. The design factors of pure rolling movable teeth transmission must ensure that there are pure rolling contact characteristics between meshing components. According to the principle of sliding and rolling substitution, the local degree of freedom is formed in the composition of two higher pair elements, and the relative sliding phenomenon is eliminated. The most effective method of mechanism design is to replace the integral type movable teeth with the combined

${ }^{\mathrm{a} C}$ Corresponding author: yiyali@ysu.edu.cn

(C) 2016. The authors - Published by Atlantis Press 
movable teeth that is made up with movable teeth axis 4 , needle roller bearing 5 and needle roller bearing 6 , and is distributed in movable teeth cogging 3.Through needle roller bearing, the local degree of freedom is formed in the composition of two higher pair elements. Pure rolling contact meshing could be realized between the teeth profile of wave cam and center wheel, between movable teeth cogging and movable tooth. For example, center wheel is fixed, wave cam is input and active tooth frame is output. When wave cam rotates at a constant speed, the movable teeth center is always at the point where the theoretical profiles of wave cam and center wheel intersect and rotate around the axis. As output, active tooth frame is pushed to rotate. According to a definite transmission ratio, the teeth profile of wave cam and center wheel is insured. The active tooth frame is output with the corresponding reduction ratio. The principle of pure rolling movable tooth transmission is shown in Figure 1.

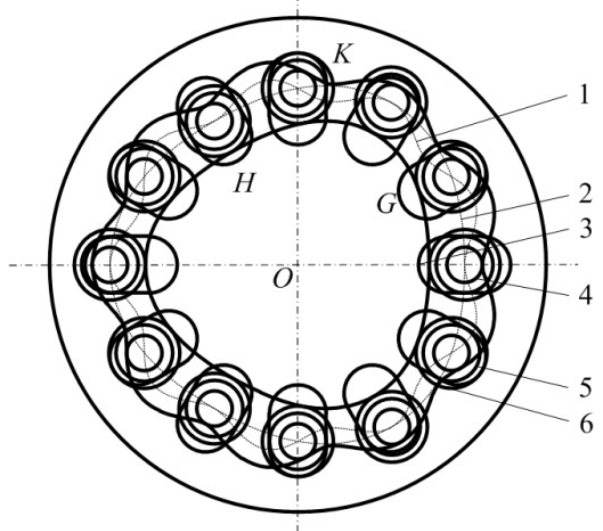

Figure 1. Principle of pure rolling movable teeth transmission

\section{The dynamic model for pure rolling movable teeth transmission}

The force analysis of movable teeth in any push travel meshing area is performed. The shock wave cam rotates with the angular velocity and turn The normal pressure of the shock wave cam to the movable teeth is , and the angle between the direction of the force and the $\mathrm{X}$ axis is ; The normal pressure of the center wheel to the movable teeth is , and the angle between the direction of the force and the $\mathrm{X}$ axis is .The normal pressure of the active teeth frame to the movable teeth is ,and the angle between the direction of the force and the $\mathrm{X}$ axis is , shown in Figure 2.

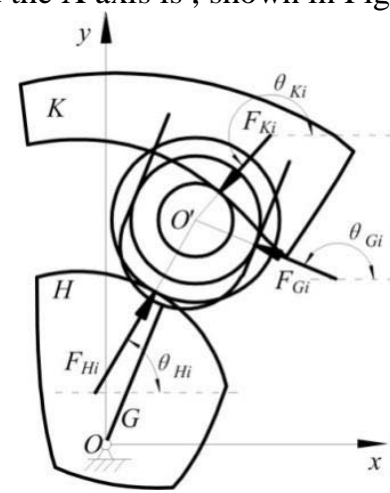

Figure 2. Rolling movable teeth transmission force diagram

Rolling movable teeth transmission have a half of movable tooth to mesh at any time in theory. This complex over-constrained mechanism have complex stress situation. When analyzed the force, the deformation compatibility equation is considered. The method for force analysis of each level 
transmission is similar. The force analysis of the rolling movable teeth transmission is carried out by taking the three-tooth difference shock cam rolling movable teeth transmission as an example. Assuming that combined tooth and the center wheel, active tooth frame and the shock cam are small deformation, and the friction force is not considered. Three teeth difference shock wave cam is the central symmetry mechanism. When the shock wave cam rotates at the push stroke, the combined movable tooth are situated in three regions of the relative rotation centersymmetrically. In addition, the force condition of the corresponding combined movable teeth in the three meshing regions is symmetric about the center of rotation. So any push travel meshing area of combined movable teeth is selected to take stress analysis.

The force balance equations of the movable teeth are as follows

$$
\left\{\begin{array}{l}
\sum X=F_{H i} \cos \theta_{H i}+F_{K i} \cos \theta_{K i}+F_{G i} \cos \theta_{G i}-m a_{x}=0 \\
\sum Y=F_{H i} \sin \theta_{H i}+F_{K i} \sin \theta_{K i}+F_{G i} \sin \theta_{G i}-m a_{y}=0
\end{array}\right.
$$

Where is the acceleration of the movable teeth center in the $\mathrm{X}$ direction is the acceleration of the movable teeth center in the Y direction. Assuming that the input torque is, the number of movable teeth within each of push travel meshing area is $n$. Due to the sum of torque of the rotating shaft of the force $\mathrm{H}$ that is F's reaction force is equal to the input torque acting on the shock wave cam. Three push travel meshing area are central symmetry. Therefore, torque balance equation of shock wave cam is

$$
M_{O}=3 \sum_{i=1}^{n} F_{H i}^{\prime}\left|d_{i}\right| \sin \gamma_{i}
$$

Where is the distance from the center of rotation to the center of i movable teeth.

As shown in Figure 3, O is the center of rotation of the shock wave cam, is the geometric center of combined type movable teeth. There are contact deformation at three meshing point of each combined type movable teeth. Interaction of three-contact deformation could lead to shock wave camrotating a very small angle. At the contact point of the movable teeth and the shock wave cam, due to three contact deformation lead to the fact that the displacement of this point along the direction of is .At the same time displacement lead to shock wave cam rotating a very small angle. The relations of the parameters are as follows

$$
\left\{\begin{array}{l}
\xi_{1 i}=\delta_{H i}+\delta_{K i} \cos \left(\pi+\theta_{K i}-\theta_{H i}\right)+\delta_{G i} \cos \left(\pi / 2-\gamma_{i}\right) \\
\xi_{1 i}=\xi_{2 i} \\
\xi_{2 i}=\Delta \varphi_{i}\left|d_{i}\right| \sin \gamma_{i}
\end{array}\right.
$$

Where are respectively normal contact deformation of the shock wave cam, the central wheel and the movable tooth frame. $\mathrm{i}=1,2, \ldots, \mathrm{n}$.

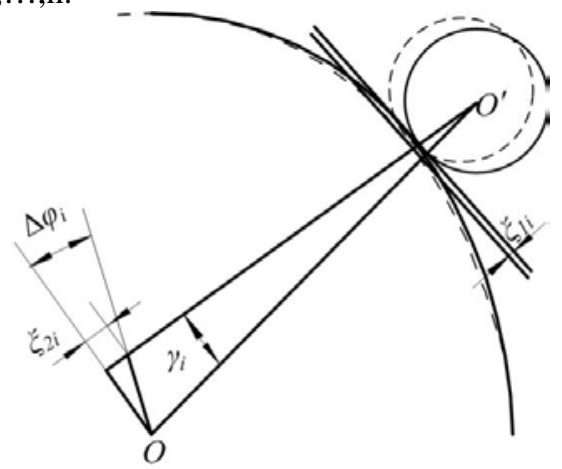

Figure 3.The deformation coordination diagram of the movable teeth meshing pair 
According to the deformation compatibility condition, this small angle that the contact deformation of each movable tooth in the meshing state lead to the shock wave cam to rotate is the same at the instant of each meshing. $=+1(i=1,2, \ldots, n-1)$. Therefore, the deformation coordination relationship of two adjacent movable teeth at the same meshing state could be obtained.

$$
\frac{\left|d_{i}\right| \sin \gamma_{i}}{\left|d_{i+1}\right| \sin r_{i+1}}=\frac{\delta_{H i}+\delta_{K i} \cos \left(\pi+\theta_{K i}-\theta_{H i}\right)+\delta_{G i} \cos \left(\pi / 2-\gamma_{i}\right)}{\delta_{H i+1}+\delta_{K i+1} \cos \left(\pi+\theta_{K i+1}-\theta_{H i+1}\right)+\delta_{G i+1} \cos \left(\pi / 2-\gamma_{i+1}\right)}
$$

The relationship between meshing force and contact deformation of the combined movable teeth meshing pair could be obtained. They are correlated variable.

The relationship between meshing force and contact deformation of shock wave cam, central wheel, movable tooth frame, combined type movable teeth are as follows

$$
\left\{\begin{array}{c}
F_{H i}=\frac{4 E \sqrt{\rho_{H} r_{1} /\left(\phi_{H}+r_{1}\right.}}{3} \delta_{H i} \\
F_{K i}=\frac{4 E \sqrt{\rho_{K} r_{2} /\left(\rho_{K} \pm r_{2}\right.}}{3} \delta_{K i} \\
F_{G i}=\frac{4 E \sqrt{\gamma_{3}}}{3} \delta_{G i}
\end{array}\right.
$$

Where are the curvature radius of the shock wave cam and the central wheel at the engaging position, respectively; are the curvature radius of the shock wave cam, the central wheel and combined movable teeth at the contact position. Positive sign express outer contact and negative sign express inner contact.

The main structure parameters of the selected rolling movable teeth transmission are: base circle radius $\mathrm{R}=40 \mathrm{~mm}$, lift $\mathrm{h}=4 \mathrm{~mm}$, movable teeth axis radius $=3 \mathrm{~mm}$, combined movable teeth mesh width $\mathrm{B}=12 \mathrm{~mm}$, meshing teeth number $=12$, shock wave cam teeth number $=3$, center wheel teeth number $=9$, transmission ratio $i=4$. Elastic modulus of materials $E=210 \mathrm{Gpa}$, Poisson ratio $\mathrm{u}=0.3$, input torque $=20 \mathrm{Nm}$. According to the formula (1) to (5), using the solving equations of movable teeth meshing force solved by MATLAB programming, the meshing force curve of movable teeth could be obtained. As shown in Figure4.

represent meshing force change with relative rotation angle of movable teeth to shock wave cam between shock wave cam and movable teeth; represent meshing force change with relative rotation angle of movable teeth to shock wave cam between central wheel and movable teeth; represent meshing force change with relative rotation angle of movable teeth to shock wave cam between movable teeth frame and movable teeth; In the process of combined movable tooth from meshing to exit, meshing force with relative rotation angle of movable teeth to shock wave cam change constant between shock wave cam and movable teeth, between central wheel and movable teeth and between movable teeth frame and movable teeth. Changing from zero to large, then became stable, and then gradually reducing to zero, overall change is flat. The meshing force between central wheel and movable teeth is the maximum, which between shock wave cam and movable teeth is followed, which between movable teeth frame and movable teeth is the minimum. 


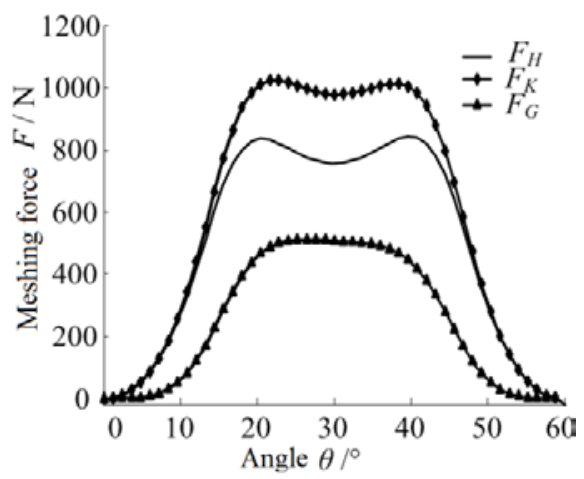

Figure 4.Curves of meshing force between movable teeth meshing pairs

\section{The manufacture of prototype}

According to the above research, the prototype of pure rolling movable teeth transmission was manufactured, shown in Figure 5.

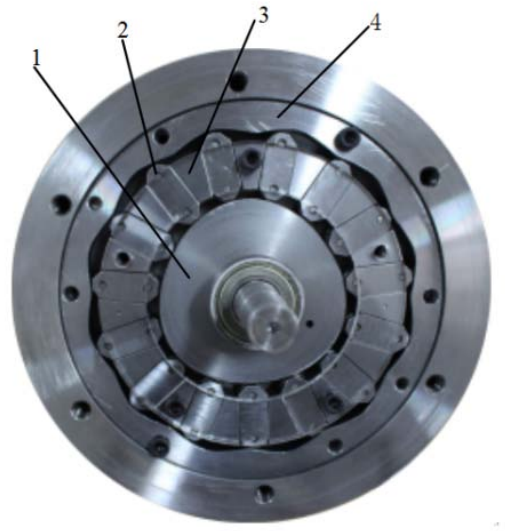

Figure 5. Prototype of pure rolling movable teeth transmission

1 is shock wave cam, 2 is movable teeth, 3is movable teeth carrier, 4 is center wheel. The prototype operates well without teeth profile interference.

\section{Summary}

According to the principle of sliding and rolling substitution, a combined type of movable teeth is designed and pure rolling movable transmission is realized. Based on the elastic deformation coordination condition, the force mathematical model of meshing pairs of pure rolling movable teeth transmission is established. The variation trend of meshing force between the movable teeth and shock wave cam, center wheel and movable teeth is obtained using the MATLAB programming. Meshing position of maximum meshing force in the transmission process is ensured. A prototype is manufactured and some experiments will be conducted in the future.

\section{Acknowledgement}

This material is based upon work supported by Science and Technology Research Youth Found Project of Hebei Province( No.QN2016095), and Independent Research Programs for Young Teachers 
of Yanshan University(No.15LGA004).

\section{References}

1. Y.L. Yi, P.P. Liu,Z.J. An,Research on tooth profile of high-order polynomial curve of arbitrary tooth difference push rod movable teeth, J.Mech.Trans., 38 (2014) 1-4.

2. J.Z. Huang, S.G.Ching, X.X. Chen, Arbitrary tooth difference of pure rolling movable teeth transmission,Mach.Des.\& Res.,24 (2008) 44-46.

3. S.Y.Zhou, Y.Y.Zeng, X.M.Yuan, Study on stiffness of torsional vibration system of cylinder sine oscillation tooth transmission, Chn. Mech. Eng., 20(2016)2711-2715,2720

4. X.R. Dong, J.F. Li, X.H.Wang,Analysis on the structure and profile of the cam shock type movable teeth transmission, Chin. Mech.Eng.,17 (2006) 1661-1665.

5. X. Bai,S.Wang, S.X. Chen,Meshing force and contact stress analysis on discrete tooth harmonic drive, J.Chongqing Univ.,36 (2013) 35-42.

6. Hidetsugu TERADA, Kenji IMASE. Fundamental analysis of a cycloid ball reducer (5th report)-Development of a two stage type reduction mechanism. JSPE, 75 (2009) 1418-1422.

7. D.W.Liu, T.Z.Ren, Y.L.Zhang, Transmission theory and typical structure of nonuniform mechanism with movable teeth, J.Mech.Eng., 1(2014)47-54

8. D.W.Liu, T.Z.Ren, Q.Ma, Design theory of motion integration mechanism with roller movable tooth,Chn. Mech. Eng.,24(2015)3318-3322,3335 